A Platinum Open Access Journal for Organic Chemistry

Paper

Free to Authors and Readers

DOAJ Seal

Arkivoc 2021, part viii, 77-85

\title{
Synthesis of furano[2,3-g]indoles from activated indoles
}

\author{
Karin Pchalek, Naresh Kumar, and David StC. Black* \\ School of Chemistry, UNSW Sydney, Sydney, NSW 2052, Australia \\ Email: d.black@unsw.edu.au
}

Received 01-11-2021

Accepted 02-16-2021

Published on line $02-19-2021$

\section{Abstract}

3-(4-Chlorophenyl)-6-hydroxy-4-methoxy-1-(toluene-4-sulfonyl)indole-7-carbaldehyde undergoes alkylation at the phenolic $\mathrm{OH}$ group with $\alpha$-haloketones to give the corresponding ethers which can be cyclised with further base treatment to give $\mathrm{N}$-tosylated furano[2,3-g]indoles. The indole $\mathrm{NH}$ can then be deprotected using potassium hydroxide in methanol to give furano[2,3-g] indoles.<smiles>[R]c1cn([18OH])c2c(C)c(O)cc(OC)c12</smiles>

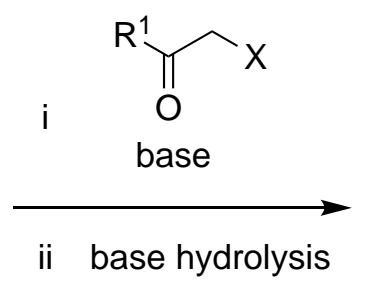

3 examples - high yields<smiles>[R]C(=O)c1cc2c(cc(OC)c3c([2H])c[nH]c32)o1</smiles>

Keywords: indoles, carbaldehydes, furan synthesis, phenols, alkylation, cyclisation 


\section{Introduction}

Tricyclic structures such as $2 \mathrm{H}$-furano[3,2-f]indoles $\mathbf{1}$ and $1 \mathrm{H}$-furano[2,3-g]indoles $\mathbf{2}$ are not known, but are structurally closely related to the tetracyclic benzo[b]furanoindoles $\mathbf{3}$ and $\mathbf{4}$, as well as the indolobenzofurans $\mathbf{5}$ and furanocarbazole alkaloids of type 6 (Figure 1).

Compounds of type $\mathbf{3}$ are important antitumor agents against certain human breast cancer cells, epidermoid carcinoma cells and melanoma cells, and show low toxicity against normal cells. ${ }^{1,2}$ On the other hand, compounds of type $\mathbf{5}$ and $\mathbf{6}$ can be found in nature and are important alkaloids due to their pharmacological potential. ${ }^{3-12}$<smiles>c1cc2cc3ccoc3cc2[nH]1</smiles>

1

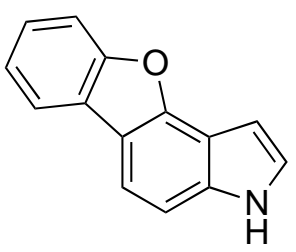

4<smiles>c1cc2ccc3occc3c2[nH]1</smiles>

2<smiles>c1ccc2c(c1)oc1ccc3cc[nH]c3c12</smiles>

5<smiles>c1ccc2c(c1)oc1cc3[nH]ccc3cc12</smiles>

3<smiles>c1ccc2c(c1)[nH]c1c3ccoc3ccc21</smiles>

6

Figure 1. Some tri- and tetra-furanoindole structures 1-6.

We have recently reported ${ }^{13}$ the preparation of the 6-hydroxy-4-methoxyindole-7-carbaldehyde 7, and consequently the possible synthesis of examples of the novel tricyclic systems $\mathbf{1}$ and $\mathbf{2}$ was investigated (Scheme 1). While reaction at C5 is not usually observed in 4,6-dimethoxyindoles, it can occur in the less well protected 4-methoxy-6-hydroxyindoles. Several aspects of the following work have been mentioned in the report of a conference lecture. ${ }^{14}$<smiles>c1cc2cc3ccoc3cc2[nH]1</smiles>

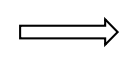

1<smiles>[R]c1cn([13C]([O-])([O-])[O-])c2c(C=O)c(O)cc(OC)c12</smiles>

$7 \mathrm{R}=4-\mathrm{ClC}_{6} \mathrm{H}_{4}$

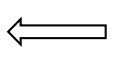<smiles>c1cc2ccc3occc3c2[nH]1</smiles>

2

Scheme 1. Possible formation of furanoindoles from 6-hydroxyindole-7-carbaldehyde 7. 


\section{Results and Discussion}

\section{Possible reaction paths}

The benzofuran ring system, which is a substructure of the tricyclic structures $\mathbf{1}$ and $\mathbf{2}$, can be synthesized from salicylaldehydes or activated phenols via $\alpha$-aryloxyketones in two steps. ${ }^{15,16}$ The successful application of those reactions to the 6-hydroxyindole $\mathbf{7}$ would possibly give derivatives of the heterocyclic scaffolds $\mathbf{1}$ and $\mathbf{2}$ (Scheme 1).

\section{Base catalyzed alkylation and cyclisation}

In order to avoid alkylation of the indole $\mathrm{NH}$, the $\mathrm{N}$-tosyl-protected 6-hydroxyindole 7 was chosen over a related unprotected indole. Alkylation of indole 7 with $\alpha$-haloketones or esters would provide indolo-ethers, which could then potentially undergo intramolecular cyclisation to give derivatives of either the furano[2,3g] indole 2 or the furano[3,2-f]indole 1.

Preliminary experiments showed that the best results were obtained with potassium carbonate in boiling acetone. Other reaction conditions, such as sodium bicarbonate in acetone, potassium carbonate in ethanol, or sodium hydride in tetrahydrofuran, gave rather poor conversions or yielded product mixtures. Consequently, reaction of indole 7 with ethyl bromoacetate gave the indole ether $\mathbf{8}$ in $68 \%$ yield. A similar reaction with 2-bromopropiophenone gave the indole ether 9 in $62 \%$ yield. On the other hand, reaction of indole 7 with 2-bromoacetophenone and 2-bromo-4'-chloroacetophenone gave the furanoindoles 13 and 14 respectively, presumably via unstable indole ethers 10 and 11 respectively. The ether 10 could be isolated using a shorter reaction time, but was unstable and underwent rapid cyclisation to the furanoindole 13: the ether 11 could not be isolated. Reaction of indole 7 with chloroacetone simply gave the furanoindole 15, without any isolation or detection of the presumed intermediate $\mathbf{1 2}$ (Scheme 2).

Attempts to extend this cyclisation process to the indole ester $\mathbf{8}$ failed. The use of stronger bases, such as sodium ethoxide or sodium hydroxide, did not afford the cyclised product, but instead yielded the related free carboxylic acid $\mathbf{1 7}$ after work-up. Furthermore, base-catalysed cyclisation attempts with indole ether $\mathbf{9}$ resulted only in recovered starting material, since the intermediate cyclisation product is not able to aromatise to the furan ring.

The tosyl group of the furanoindoles 13-15 could be removed easily with crushed potassium hydroxide in refluxing methanol, yielding the furanoindoles $\mathbf{1 8 - 2 0}$ respectively in $73-91 \%$ yield. The structures of these furanoindoles were confirmed by $1 \mathrm{D}$ and 2D NMR experiments.

\section{Acid-catatysed cyclisation}

On the basis that the synthesis of benzofurans can be achieved by intramolecular cyclisation from $\alpha-$ aryl(alkyl)oxyketones in the presence of trifluoroacetic acid, ${ }^{8,9}$ cyclisation of indole ethers $\mathbf{9}$ and 10 was attempted to give furano[3,2-f]indoles of type 1. However, formation of the desired furano[3,2-f]indoles was not observed: indole ether 10 gave the furano[2,3-g]indole 13, but indole ether 9 failed to react and only starting material was recovered.

\section{Reduction of the carbonyl group}

The carbonyl groups on the furanoindoles $\mathbf{1 8}$ and $\mathbf{2 0}$ were easily reduced using sodium borohydride in ethanol, giving excellent yields of the corresponding stable alcohols $\mathbf{2 2}$ and $\mathbf{2 3}$ (Scheme 2). These alcohols were of interest in that they incorporate a nucleophilic site at the indole C2 and an electrophilic site at the 
hydroxymethyl group of the furan ring. However, treatment of the alcohols $\mathbf{2 2}$ and $\mathbf{2 3}$ under a variety of acidic conditions led only to complex polymeric mixtures.

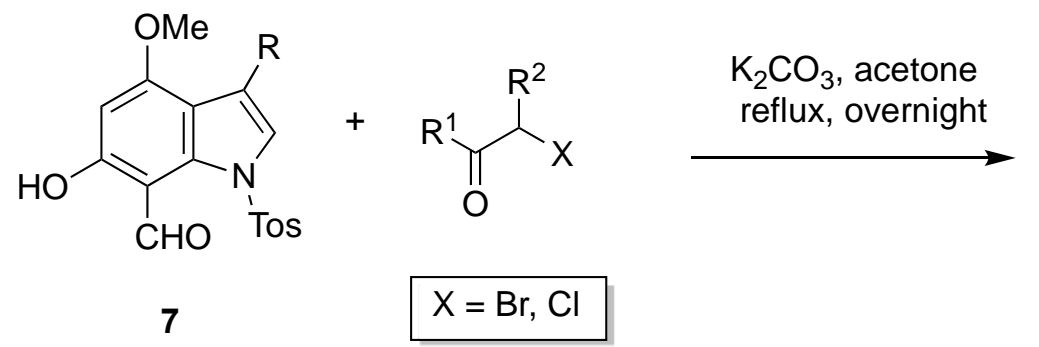

$$
\mathrm{R}=4-\mathrm{CIC}_{6} \mathrm{H}_{4}
$$

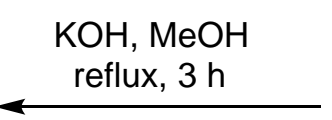

$\mathrm{NaBH}_{4}, \mathrm{EtOH}$ reflux, $1 \mathrm{~h}$<smiles>[R]c1c[nH]c2c1c(OC)cc1oc(C([R])O)cc12</smiles><smiles>[R]c1cn([18O])c2c(C)c(OCC(=O)OCC)cc(OC)c12</smiles>

8

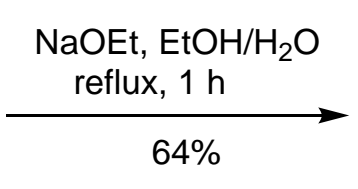

$\mathrm{NaOEt}, \mathrm{EtOH} / \mathrm{H}_{2} \mathrm{O}$ $64 \%$<smiles>[R]C(=O)C([R7])Oc1cc(OC)c2c([R])cn([AsH3])c2c1C</smiles>

\begin{tabular}{|clcc|}
\hline & $\mathrm{R}^{1}$ & $\mathrm{R}^{2}$ & \% yield \\
\hline $\mathbf{8}$ & $\mathrm{OEt}$ & $\mathrm{H}$ & 68 \\
$\mathbf{9}$ & $\mathrm{Ph}$ & $\mathrm{Me}$ & 62 \\
$\mathbf{1 0}$ & $\mathrm{Ph}$ & $\mathrm{H}$ & 93 \\
$\mathbf{1 1}$ & $4-\mathrm{ClC}_{6} \mathrm{H}_{4}$ & $\mathrm{H}$ & - \\
$\mathbf{1 2}$ & $\mathrm{Me}$ & $\mathrm{H}$ & - \\
\hline
\end{tabular}<smiles>[R]c1cn([O+])c2c1c(OC)cc1oc(C(=O)O)cc12</smiles>

\begin{tabular}{|llr|}
\hline & $\mathrm{R}^{1}$ & \% \\
\hline 13 & $\mathrm{Ph}$ & 88 \\
14 & $4-\mathrm{ClC}_{6} \mathrm{H}_{4}$ & 86 \\
15 & $\mathrm{Me}$ & 78 \\
\hline
\end{tabular}

Scheme 2. Formation of furanoindoles 18-20. 


\section{Conclusions}

The activation of indoles leads to the synthesis of a 6-hydroxy-4-methoxyindole-7-aldehyde, which in turn can be converted by reaction with suitable $\alpha$-haloketones to the previously unknown furano[2,3-g]indoles.

\section{Experimental Section}

General. Melting points were measured using a Mel-Temp melting point apparatus. Microanalyses were performed on Carlo Erba Elemental Analyser EA 1108 at the Campbell Microanalytical Laboratory, University of Otago, New Zealand. ${ }^{1} \mathrm{H}$ and ${ }^{13} \mathrm{C}$ NMR spectra were obtained on a Bruker DPX300 (300 MHz) spectrometer and internally referenced to solvent peaks. Mass spectra were recorded on either a Bruker Daltonics Bio Apex II FTICR MS (HRMS-ESI) at the School of Chemistry, University of New South Wales, or a Shimadzu LCMS QP 8000 (EI) at the Univeristy of Otago, New Zealand. Infrared spectra were recorded with a Thermo Nicolet 370 FTIR Spectrometer using $\mathrm{KBr}$ discs. Ultraviolet-visible spectra were recorded using a Varian Cary 100 Scan Spectrometer.

[3-(4-Chlorophenyl)-7-formyl-4-methoxy-1-(toluene-4-sulfonyl)indol-6-yloxy]acetic acid ethyl ester (8). A mixture of indole $7(0.20 \mathrm{~g}, 0.44 \mathrm{mmol})$, ethyl bromoacetate $(0.05 \mathrm{~mL}, 0.44 \mathrm{mmol})$ and potassium carbonate $(0.12 \mathrm{~g}, 0.75 \mathrm{mmol})$ in acetone $(20 \mathrm{~mL})$ was heated under reflux overnight. The reaction mixture was diluted with dichloromethane $(20 \mathrm{~mL})$, washed with saturated aqueous ammonium chloride $(2 \times 10 \mathrm{~mL}), \mathrm{water}(10 \mathrm{~mL})$ and brine $(10 \mathrm{~mL})$, and dried over $\mathrm{MgSO}_{4}$. The solvent was evaporated and the product purified by column chromatography on silica gel $\left(\mathrm{CH}_{2} \mathrm{Cl}_{2} / \mathrm{MeOH}, 98: 2\right)$ yielding indole 8 as an off-white solid $(0.16 \mathrm{~g}, 0.30 \mathrm{mmol}$, 68\%), $\mathrm{mp}(\mathrm{MeOH}) 59-61{ }^{\circ} \mathrm{C} . \mathrm{IR}\left(\mathrm{v} \max , \mathrm{cm}^{-1}\right): 3419,2980,1755,1693,1590,1369,1199,1171,1089,812,666$, 543. UV/Vis $\left(\lambda \max , \mathrm{nm}, \varepsilon, \mathrm{cm}^{-1} \mathrm{M}^{-1}\right): 246(37,100), 296(12,900) .{ }^{1} \mathrm{H} N M R\left(300 \mathrm{MHz}, \mathrm{CDCl}_{3}\right): \delta_{\mathrm{H}} 1.29(3 \mathrm{H}, \mathrm{t}, J 7.1$ $\left.\mathrm{Hz}, \mathrm{CH}_{2} \underline{\mathrm{Me}}\right), 2.36(3 \mathrm{H}, \mathrm{s}, \mathrm{Me}), 3.74(3 \mathrm{H}, \mathrm{s}, \mathrm{OMe}), 4.26\left(2 \mathrm{H}, \mathrm{q}, J 7.1 \mathrm{~Hz}, \mathrm{CH}_{2} \mathrm{Me}\right), 4.80\left(2 \mathrm{H}, \mathrm{s}, \mathrm{CH}_{2}\right), 6.41(1 \mathrm{H}, \mathrm{s}$, H5), $7.20(2 \mathrm{H}, \mathrm{d}, J 8.3 \mathrm{~Hz}, \operatorname{aryl}), 7.32(4 \mathrm{H}, \mathrm{br}$ s, aryl), $7.40(1 \mathrm{H}, \mathrm{s}, \mathrm{H} 2), 7.57(2 \mathrm{H}, \mathrm{d}, J 8.7 \mathrm{~Hz}, \operatorname{aryl}), 10.36(1 \mathrm{H}, \mathrm{s}$, $\mathrm{CHO}) .{ }^{13} \mathrm{C} \mathrm{NMR}\left(75 \mathrm{MHz}, \mathrm{CDCl}_{3}\right): \delta \mathrm{c} 14.1\left(\mathrm{CH}_{2} \mathrm{Me}\right), 21.5(\mathrm{Me}), 55.4(\mathrm{OMe}), 61.4\left(\mathrm{CH}_{2}\right), 69.1\left(\mathrm{CH}_{2}\right), 95.5(\mathrm{C} 5)$, 111.4, 116.0 and 125.0 (aryl C), 126.6 (C2), 126.9, 128.7, 129.5 and 130.6 (aryl CH), 131.6, 133.4, 134.5, 137.2, 145.0, 157.6 and 158.4 (aryl C), 168.5 (CO), 187.0 (CHO). MS (+EI, m/z, \%): 543 (42, $\mathrm{MH}^{+}$), 388 (100). Anal. calcd for $\mathrm{C}_{27} \mathrm{H}_{24} \mathrm{ClNO}_{7} \mathrm{~S}$ : C, 59.83; $\mathrm{H}, 4.46 ; \mathrm{N}, 2.58$. Found: $\mathrm{C}, 59.67 ; \mathrm{H}, 4.26 ; \mathrm{N}, 2.52 \%$.

\section{3-(4-Chlorophenyl)-4-methoxy-6-(1-methyl-2-oxo-2-phenylethoxy)-1-(toluene-4-sulfonyl)indole-7-}

carbaldehyde (9). A mixture of indole $7(0.25 \mathrm{~g}, 0.55 \mathrm{mmol}), 2$-bromopropiophenone $(0.1 \mathrm{~mL}, 0.55 \mathrm{mmol})$ and potassium carbonate $(0.13 \mathrm{~g}, 0.93 \mathrm{mmol})$ in acetone $(25 \mathrm{~mL})$ was heated under reflux overnight. The mixture was diluted with dichloromethane $(25 \mathrm{~mL})$ and washed with both aqueous $\mathrm{HCl}(2 \mathrm{M}, 2 \times 25 \mathrm{~mL})$ and brine $(2 \times 25$ $\mathrm{mL}$ ) after which the organic layer was dried with $\mathrm{Na}_{2} \mathrm{SO}_{4}$ and the solvent removed under reduced pressure. The crude product was purified by column chromatography on silica gel $\left(\mathrm{CH}_{2} \mathrm{Cl}_{2} / \mathrm{MeOH}, 98: 2\right)$ yielding indole 9 as an off-white solid $(0.20 \mathrm{~g}, 0.34 \mathrm{mmol}, 62 \%), \mathrm{mp} \mathrm{78-82}{ }^{\circ} \mathrm{C}$. IR ( $\mathrm{v}$ max, $\left.\mathrm{cm}^{-1}\right): 3010,3065,2937,1694,1590$, $1369,1171,1089,811,703,543$. UV/Vis $\left(\lambda \max , \mathrm{nm}, \varepsilon, \mathrm{cm}^{-1} \mathrm{M}^{-1}\right): 248(44,700) .{ }^{1} \mathrm{H} \mathrm{NMR}\left(300 \mathrm{MHz}, \mathrm{CDCl}_{3}\right): \delta_{H}$ $1.83(3 \mathrm{H}$, br s, CHMe), $2.29(3 \mathrm{H}, \mathrm{s}, \mathrm{Me}), 3.61(3 \mathrm{H}, \mathrm{s}, \mathrm{OMe}), 5.68(1 \mathrm{H}, \mathrm{br} \mathrm{s}, \mathrm{CHMe}), 6.39(1 \mathrm{H}, \mathrm{s}, \mathrm{H} 5), 7.01-8.12$ $\left(14 \mathrm{H}, \mathrm{m}, \mathrm{H} 2\right.$ and aryl), $10.34(1 \mathrm{H}, \mathrm{s}, \mathrm{CHO}) .{ }^{13} \mathrm{C} \mathrm{NMR}$ (75 MHz, DMSO-d $)$ : $\delta 19.0$ (CHMe), 21.5 (Me), $55.3(\mathrm{OMe})$, 81.5 (CHMe), 96.7 (C5), 111.2, 115.8 and 125.6 (aryl C), 126.5 (C2), 126.8, 127.7, 128.8, 128.9, 129.3 and 130.5 (aryl CH), 131.4 and 133.4 (aryl C), 133.8 (aryl CH), 133.9, 134.1, 138.0, 144.9, 157.7 and 157.9 (aryl C), 186.6 
(CHO), 199.0 (CO). MS (+El, m/z, \%): 644 (19), 589 (32, $\left.\mathrm{MH}^{+}\right), 434$ (100), 312 (46), 301 (30). Anal. calcd for $\mathrm{C}_{32} \mathrm{H}_{26} \mathrm{ClNO}_{6} \mathrm{~S} .0 .5 \mathrm{CH}_{2} \mathrm{Cl}_{2}$ : C, 61.91; $\mathrm{H}, 4.32 ; \mathrm{N}, 2.22$. Found: $\mathrm{C}, 61.88 ; \mathrm{H}, 4.25 ; \mathrm{N}, 2.22 \%$.

3-(4-Chlorophenyl)-4-methoxy-6-(2-oxo-2-phenylethoxy)-1-(toluene-4-sulfonyl)indole-7-carbaldehyde (10). A mixture of indole 7 (0.35 g, $0.77 \mathrm{mmol}), 2$-bromoacetophenone $(0.15 \mathrm{~g}, 0.77 \mathrm{mmol})$ and potassium carbonate $(0.18 \mathrm{~g}, 1.31 \mathrm{mmol})$ in acetone $(35 \mathrm{~mL})$ was heated under reflux overnight. The reaction mixture was diluted with aqueous $\mathrm{HCl}(2 \mathrm{M}, 40 \mathrm{~mL})$, extracted with dichloromethane $(2 \times 35 \mathrm{~mL})$, the combined organic layers washed with brine $(25 \mathrm{~mL})$ and dried over $\mathrm{Na}_{2} \mathrm{SO}_{4}$. The solvent was evaporated and the product purified by column chromatography on silica gel $\left(\mathrm{CH}_{2} \mathrm{Cl}_{2}\right)$ yielding indole 10 as a yellow solid $(0.41 \mathrm{~g}, 0.72 \mathrm{mmol}, 93 \%)$, $\mathrm{mp} 206-208{ }^{\circ} \mathrm{C}$. IR $\left(v \max , \mathrm{cm}^{-1}\right)$ : 3429, 1699, 1599, 1494, 1373, 1175, 1134, 1090, 813, 679. UV/Vis $(\lambda \mathrm{max}, \mathrm{nm}$, $\left.\varepsilon, \mathrm{cm}^{-1} \mathrm{M}^{-1}\right): 237(41,700), 369$ (1,500). The compound was too unstable for ${ }^{1} \mathrm{H}$ NMR spectrum measurement, because of rapid cyclisation to compound 13. MS (+EI, $m / z, \%): 573\left(20, \mathrm{M}^{+}\right), 555$ (59), 418 (100). Anal. calcd for $\mathrm{C}_{31} \mathrm{H}_{24} \mathrm{ClNO}_{6} \mathrm{~S}$ : C, 64.86; $\mathrm{H}, 4.21 ; \mathrm{N}, 2.44$. Found: C, 65.05; H, 4.16; N, 2.37\%.

[3-(4-Chlorophenyl)-4-methoxy-1-(toluene-4-sulfonyl)furano[2,3-g]indol-7-yl] phenyl ketone (13). Method A: A mixture of indole $7(0.35 \mathrm{~g}, 0.77 \mathrm{mmol}), 2$-bromoacetophenone $(0.15 \mathrm{~g}, 0.77 \mathrm{mmol})$ and potassium carbonate $(0.18 \mathrm{~g}, 1.31 \mathrm{mmol})$ in acetone $(35 \mathrm{~mL})$ was heated under reflux for $24 \mathrm{~h}$. The reaction mixture was diluted with aqueous $\mathrm{HCl}(2 \mathrm{M}, 40 \mathrm{~mL})$, extracted with dichloromethane $(2 \times 35 \mathrm{~mL})$, the combined organic layers washed with brine $(25 \mathrm{~mL})$ and dried over $\mathrm{Na}_{2} \mathrm{SO}_{4}$. The solvent was evaporated and the product purified by column chromatography on silica gel $\left(\mathrm{CH}_{2} \mathrm{Cl}_{2}\right)$ yielding compound $\mathbf{1 3}$ as a bright yellow powder $(377 \mathrm{mg}$, $0.678 \mathrm{mmol}, 88 \%), \mathrm{mp} 212-214^{\circ} \mathrm{C}$.

Method B: A solution of indole $10(100 \mathrm{mg}, 0.174 \mathrm{mmol})$ in trifluoroacetic acid $(2.5 \mathrm{~mL})$ was heated under reflux for $5 \mathrm{~h}$ and poured into ice-cold aqueous $\mathrm{NaOH}(0.2 \mathrm{M}, 5 \mathrm{~mL})$. Dichloromethane $(5 \mathrm{~mL})$ was added, the organic layer separated, dried with $\mathrm{MgSO}_{4}$, evaporated in vacuo and the resulting green solid purified by flash chromatography on silica gel $\left(\mathrm{CH}_{2} \mathrm{Cl}_{2}\right)$ yielding compound 13 as a bright yellow powder $(79 \mathrm{mg}, 0.143 \mathrm{mmol}$, $82 \%), \operatorname{mp~} 212-214{ }^{\circ} \mathrm{C}$. IR $\left(\mathrm{V} \max , \mathrm{cm}^{-1}\right)$ : 3441, 2937, 1641, 1613, 1540, 1279, 1175, 1136, 1093, 677. UV/Vis ( $\lambda$ $\left.\max , \mathrm{nm}, \varepsilon, \mathrm{cm}^{-1} \mathrm{M}^{-1}\right): 230$ (38,000), 369 (12,000). ${ }^{1} \mathrm{H}$ NMR (300 MHz, CDCl 3 ): $\delta_{\mathrm{H}} 2.34(3 \mathrm{H}, \mathrm{s}, \mathrm{Me}), 3.80(3 \mathrm{H}, \mathrm{s}$, OMe), $6.98(1 \mathrm{H}, \mathrm{s}, \mathrm{H} 5), 7.22(2 \mathrm{H}, \mathrm{d}, J 7.9 \mathrm{~Hz}$, tosyl), $7.36(2 \mathrm{H}, \mathrm{d}, J 6.4 \mathrm{~Hz}, \operatorname{aryl}), 7.44(2 \mathrm{H}, \mathrm{d}, J 6.4 \mathrm{~Hz}, \operatorname{aryl}), 7.54$ $(1 \mathrm{H}, \mathrm{s}, \mathrm{H} 2), 7.55-7.70(5 \mathrm{H}, \mathrm{m}, \mathrm{aryl}), 8.02\left(2 \mathrm{H}, \mathrm{d}, J 7.9 \mathrm{~Hz}\right.$, tosyl), $8.30(1 \mathrm{H}, \mathrm{s}, \mathrm{H} 8) .{ }^{13} \mathrm{C} \mathrm{NMR}\left(75 \mathrm{MHz}, \mathrm{CDCl}_{3}\right): \delta_{\mathrm{c}}$ 21.6 (Me), 55.5 (OMe), 90.2 (C5), 101.4 and 108.4 (aryl C), 117.1 (C8), 123.6 (C2), 123.9 (aryl C), 126.7, 127.7, 128.5, 129.3 and 130.1 (aryl CH), 130.9 (aryl C), 131.0 (aryl CH), 132.1 (aryl C), 132.7 (aryl CH), 133.2, 134.9, 137.5, 145.5, 150.7, 155.7, 156.9 (aryl C), 183.6 (CO). MS (+El, m/z, \%): 555 (26, M+), 400 (100), 350 (32). Anal. calcd for $\mathrm{C}_{31} \mathrm{H}_{22} \mathrm{ClNO}_{5} \mathrm{~S} .0 .5 \mathrm{CH}_{2} \mathrm{Cl}_{2}$ : C, 63.22; $\mathrm{H}, 3.87 ; \mathrm{N}, 2.34$. Found: C, 63.52; $\mathrm{H}, 3.96 ; \mathrm{N}, 2.30 \%$.

(4-Chlorophenyl) [3-(4-chlorophenyl)-4-methoxy-1-(toluene-4-sulfonyl)furano[2,3-g]indol-7-yl] ketone (14).

A mixture of indole $7(0.25 \mathrm{~g}, 0.55 \mathrm{mmol}), 2$-bromo-4-chloroacetophenone $(0.13 \mathrm{~g}, 0.55 \mathrm{mmol})$ and potassium carbonate $(0.13 \mathrm{~g}, 0.93 \mathrm{mmol})$ in acetone $(25 \mathrm{~mL})$ was heated under reflux overnight. The mixture was diluted with dichloromethane $(25 \mathrm{~mL})$ and washed with both aqueous $\mathrm{HCl}(2 \mathrm{M}, 2 \times 25 \mathrm{~mL})$ and brine $(2 \times 25 \mathrm{~mL})$, the organic layer dried with $\mathrm{Na}_{2} \mathrm{SO}_{4}$ and the solvent removed under reduced pressure. The crude product was purified by column chromatography on silica gel $\left(\mathrm{CH}_{2} \mathrm{Cl}_{2} /\right.$ light petroleum, 1:1) yielding compound 14 as a bright yellow powder (0.28 g, $0.47 \mathrm{mmol}, 86 \%), \mathrm{mp} 236-238^{\circ} \mathrm{C}$. IR ( $\left.\mathrm{v} \max , \mathrm{cm}^{-1}\right): 3119,2937,2831,1646,1611$, $1490,1375,1279,1176,1091,1014,816,677,544$. UV/Vis $\left(\lambda \max , \mathrm{nm}, \varepsilon, \mathrm{cm}^{-1} \mathrm{M}^{-1}\right): 232(42,700), 267(25,100)$, $374(20,900) .{ }^{1} \mathrm{H}$ NMR $\left(300 \mathrm{MHz}, \mathrm{DMSO}-d_{6}\right): \delta_{\mathrm{H}} 2.30(3 \mathrm{H}, \mathrm{s}, \mathrm{Me}), 3.79(3 \mathrm{H}, \mathrm{s}, \mathrm{OMe}), 7.34(1 \mathrm{H}, \mathrm{s}, \mathrm{H} 5), 7.41(2 \mathrm{H}$, d, J $8.3 \mathrm{~Hz}, \operatorname{aryl}), 7.44(2 \mathrm{H}, \mathrm{d}, J 8.3 \mathrm{~Hz}, \operatorname{aryl}), 7.55(2 \mathrm{H}, \mathrm{d}, J 8.3 \mathrm{~Hz}, \operatorname{aryl}), 7.74(2 \mathrm{H}, \mathrm{d}, J 8.3 \mathrm{~Hz}, \operatorname{aryl}), 7.79(2 \mathrm{H}, \mathrm{d}, J$ $8.7 \mathrm{~Hz}, \operatorname{aryl}), 7.84(1 \mathrm{H}, \mathrm{s}, \mathrm{H} 2), 7.99\left(2 \mathrm{H}, \mathrm{d}, J 8.7 \mathrm{~Hz}\right.$, aryl), $8.15(1 \mathrm{H}, \mathrm{s}, \mathrm{H} 8) .{ }^{13} \mathrm{C} \mathrm{NMR}\left(75 \mathrm{MHz}, \mathrm{DMSO}-d_{6}\right): \delta_{\mathrm{c}} 21.4$ (Me), 56.4 (OMe), 60.6 and 72.7 (aryl C), 91.6 (C5), 107.8 (aryl C), 116.5 (C8), 123.6 (aryl C), 124.8 (C2), 127.1 and 127.9 (aryl CH), 128.0 (aryl C), 129.3, 131.0, 131.2 and 132.0 (aryl CH), 132.4, 134.2, 136.1, 138.3, 146.5, 
150.7, 156.1 and 156.9 (aryl C), 181.6 (CO). MS (+EI, m/z, \%): 591 (95, $\mathrm{MH}^{+}$), 437 (100). Anal. calcd for $\mathrm{C}_{31} \mathrm{H}_{21} \mathrm{Cl}_{2} \mathrm{NO}_{5} \mathrm{~S}: \mathrm{C}, 63.06 ; \mathrm{H}, 3.58 ; \mathrm{N}, 2.37$. Found: $\mathrm{C}, 62.81 ; \mathrm{H}, 3.53 ; \mathrm{N}, 2.38 \%$.

1-[3-(4-Chlorophenyl)-4-methoxy-1-(toluene-4-sulfonyl)furano[2,3-g]-indol-7-yl]ethanone (15). A mixture of indole $7(0.25 \mathrm{~g}, 0.55 \mathrm{mmol})$, chloroacetone $(44 \mu \mathrm{L}, 0.55 \mathrm{mmol})$ and potassium carbonate $(0.13 \mathrm{~g}, 0.93 \mathrm{mmol})$ in acetone $(25 \mathrm{~mL})$ was heated under reflux overnight. The mixture was diluted with dichloromethane $(50 \mathrm{~mL})$ and washed both with aqueous $\mathrm{HCl}(2 \mathrm{M}, 2 \times 25 \mathrm{~mL})$ and brine $(2 \times 25 \mathrm{~mL})$, the organic layer dried with $\mathrm{Na}_{2} \mathrm{SO}_{4}$ and the solvent removed under reduced pressure. The crude product was purified by column chromatography on silica gel $\left(\mathrm{CH}_{2} \mathrm{Cl}_{2}\right)$ yielding compound 15 as a white powder $(0.21 \mathrm{mg}, 0.43 \mathrm{mmol}, 78 \%), \mathrm{mp} 236-238{ }^{\circ} \mathrm{C}$.

IR ( $\left.\mathrm{v} \max , \mathrm{cm}^{-1}\right)$ : 3437, 3137, 1667, 1611, 1493, 1372, 1276, 1179, 1142, 1093, 812, 680, 597. UV/Vis $(\lambda \max$, $\left.\mathrm{nm}, \varepsilon, \mathrm{cm}^{-1} \mathrm{M}^{-1}\right): 232(44,700), 312(20,000), 348(20,100) .{ }^{1} \mathrm{H}$ NMR $\left(300 \mathrm{MHz}, \mathrm{CDCl}_{3}\right): \delta_{\mathrm{H}} 2.34(3 \mathrm{H}, \mathrm{s}, \mathrm{Me}), 2.62$ $(3 \mathrm{H}, \mathrm{s}, \mathrm{Me}), 3.77(3 \mathrm{H}, \mathrm{s}, \mathrm{OMe}), 6.91(1 \mathrm{H}, \mathrm{s}, \mathrm{H} 5), 7.21(2 \mathrm{H}, \mathrm{d}, J 8.3 \mathrm{~Hz}, \operatorname{aryl}), 7.34(2 \mathrm{H}, \mathrm{d}, J 8.7 \mathrm{~Hz}, \operatorname{aryl}), 7.41(2 \mathrm{H}$, d, J $8.7 \mathrm{~Hz}$, aryl), $7.51(1 \mathrm{H}, \mathrm{s}, \mathrm{H} 2), 7.68\left(2 \mathrm{H}, \mathrm{d}, J 8.3 \mathrm{~Hz}\right.$, aryl), $8.34(1 \mathrm{H}, \mathrm{s}, \mathrm{H} 8) .{ }^{13} \mathrm{C} \mathrm{NMR}\left(75 \mathrm{MHz}, \mathrm{DMSO}-d_{6}\right): \delta \mathrm{c}$ 21.4 (Me), 26.7 (Me), 56.3 (OMe), 91.5 (C5), 107.8 (aryl C), 113.2 (C8), 116.3 and 123.6 (aryl C), 124.7 (C2), 127.2 and 127.8 (aryl CH), 128.1 (aryl C), 130.9 and 132.0 (aryl CH), 132.4, 132.4, 134.2, 146.4, 151.4, 155.6, 156.2 (aryl C), 187.2 (CO). MS (+El, $m / z, \%): 495$ (100, $\mathrm{MH}^{+}$), 340 (42). Anal. calcd for $\mathrm{C}_{26} \mathrm{H}_{20} \mathrm{CINO}_{5} \mathrm{~S} .0 .25 \mathrm{H}_{2} \mathrm{O}: \mathrm{C}_{\text {, }}$ $62.65 ; \mathrm{H}, 4.15 ; \mathrm{N}, 2.81$. Found: C, 62.52; H, 4.02; N, 2.81\%.

[3-(4-Chlorophenyl)-7-formyl-4-methoxyindol-6-yloxy]acetic acid (17). A solution of indole 8 (0.15 g, 0.28 $\mathrm{mmol}$ ) in ethanolic sodium ethoxide $(12.5 \%, 5 \mathrm{~mL})$ was heated under reflux for $1 \mathrm{~h}$, the suspension concentrated and the precipitate filtered off, washed with water until rinsings were neutral yielding indole 17 as a pale pink solid $(0.64 \mathrm{~g}, 0.18 \mathrm{mmol}, 64 \%), \mathrm{mp}\left(\mathrm{CHCl}_{3}\right)>300{ }^{\circ} \mathrm{C}$. IR $\left(\mathrm{v} \mathrm{max}, \mathrm{cm}^{-1}\right): 3419,2939,1629,1592$, 1318, 1273, 1207, 1095, 935, 838, 791. UV/Vis $\left(\lambda \max , \mathrm{nm}, \varepsilon, \mathrm{cm}^{-1} \mathrm{M}^{-1}\right): 252(20,400), 328(11,200) .{ }^{1} \mathrm{H} N M R$ (300 MHz, DMSO-d $): \delta_{\text {H }} 3.82(3 \mathrm{H}, \mathrm{s}, \mathrm{OMe}), 4.33\left(2 \mathrm{H}, \mathrm{s}, \mathrm{CH}_{2}\right), 6.30(1 \mathrm{H}, \mathrm{s}, \mathrm{H} 5), 7.13(1 \mathrm{H}, \mathrm{d}, J 1.9 \mathrm{~Hz}, \mathrm{H} 2), 7.35$ $(2 \mathrm{H}, \mathrm{d}, J 8.6 \mathrm{~Hz}, \operatorname{aryl}), 7.50(2 \mathrm{H}, \mathrm{d}, J 8.6 \mathrm{~Hz}, \operatorname{aryl}), 10.36(1 \mathrm{H}, \mathrm{s}, \mathrm{CHO}), 11.35(1 \mathrm{H}, \mathrm{br} \mathrm{s}, \mathrm{NH}) .{ }^{13} \mathrm{C} \mathrm{NMR}(75 \mathrm{MHz}$, DMSO- $d_{6}$ ): $\delta$ c 55.7 (OMe), $69.9\left(\mathrm{CH}_{2}\right), 89.7$ (C5), 104.8 and 116.3 (aryl C), 123.5 (C2), 127.6 (aryl C), 127.9 (aryl $\mathrm{CH}), 130.5$ (aryl C), 131.0 (aryl CH), 134.9, 136.4, 160.7 and 163.7 (aryl C), $169.8\left(\mathrm{CO}_{2} \mathrm{H}\right), 187.8$ (CHO). MS (+El, $\mathrm{m} / \mathrm{z}, \%): 361\left(30, \mathrm{MH}^{+}\right.$), 315 (100), 299 (96), 287 (56). Anal. calcd for $\mathrm{C}_{18} \mathrm{H}_{14} \mathrm{ClNO}_{5} .0 .75 \mathrm{CHCl}_{3}$ : C, 51.20; $\mathrm{H}, 3.64$; $\mathrm{N}, 3.02$. Found: $\mathrm{C}, 51.40 ; \mathrm{H}, 3.32 ; \mathrm{N}, 3.10 \%$.

[3-(4-Chlorophenyl)-4-methoxyfuro[2,3-g]indol-7-yl] phenyl ketone (18). A mixture of indole 13 (70 mg, $0.126 \mathrm{mmol}$ ) and crushed potassium hydroxide $(140 \mathrm{mg}, 2.5 \mathrm{mmol})$ in methanol $(4 \mathrm{~mL})$ was heated under reflux for $3 \mathrm{~h}$, neutralised with acetic acid (2M) and the resulting precipitate filtered off, washed with water until rinsings were neutral and dried in vacuo yielding compound $\mathbf{1 8}$ as a bright yellow powder (37 mg, 0.092 $\mathrm{mmol}, 73 \%), \mathrm{mp}\left(\mathrm{CHCl}_{3}\right) 252-254{ }^{\circ} \mathrm{C}$. IR $\left(\mathrm{v} \max , \mathrm{cm}^{-1}\right): 3226,2997,1611,1532,1487,1280,1195,1130,485$, 723. UV/Vis $\left(\lambda \max , \mathrm{nm}, \varepsilon, \mathrm{cm}^{-1} \mathrm{M}^{-1}\right): 233(30,200), 261(13,500), 386(18,200) .{ }^{1} \mathrm{H} N M R\left(300 \mathrm{MHz}, \mathrm{CDCl}_{3}\right): \delta_{H}$ $3.89(3 \mathrm{H}, \mathrm{s}, \mathrm{OMe}), 6.86(1 \mathrm{H}, \mathrm{s}, \mathrm{H} 5), 7.13(1 \mathrm{H}, \mathrm{d}, J 2.3 \mathrm{~Hz}, \mathrm{H} 2), 7.35(2 \mathrm{H}, \mathrm{d}, J 8.3 \mathrm{~Hz}, \operatorname{aryl}), 7.50-7.65(5 \mathrm{H}, \mathrm{m}, \operatorname{aryl})$, $7.69(1 \mathrm{H}, \mathrm{s}, \mathrm{H} 8), 8.05\left(2 \mathrm{H}, \mathrm{d}, J 8.3 \mathrm{~Hz}\right.$, aryl), $8.70\left(1 \mathrm{H}\right.$, br s, NH). ${ }^{13} \mathrm{C} \mathrm{NMR}\left(75 \mathrm{MHz}, \mathrm{CDCl}_{3}\right): \delta$ c 56.0 (OMe), 86.7 (C5), 107.8, 112.0, 116.4 and 117.9 (aryl C), 123.0 (C8), 127.8 (C2), 129.0 and 129.2 (aryl CH), 130.3 (aryl C), 130.6, 131.4 and 132.8 (aryl CH), 135.2, 138.1, 150.1, 155.9 and 157.2 (aryl C), 182.3 (CO). MS (+El, $m / z, \%)$ : $401\left(90, \mathrm{M}^{+}\right), 351$ (100). Anal. calcd for $\mathrm{C}_{24} \mathrm{H}_{16} \mathrm{ClNO}_{3} .0 .25 \mathrm{CHCl}_{3}: \mathrm{C}, 67.47 ; \mathrm{H}, 3.79 ; \mathrm{N}, 3.24$. Found: $\mathrm{C}, 67.74 ; \mathrm{H}$, $3.82 ; \mathrm{N}, 3.20 \%$.

(4-Chlorophenyl) [3-(4-chlorophenyl)-4-methoxyfurano[2,3-g]indol-7-yl] ketone (19). A mixture of indole 14 (100 mg, $0.169 \mathrm{mmol}$ ) and crushed potassium hydroxide (190 mg, $3.4 \mathrm{mmol}$ ) in methanol $(5 \mathrm{~mL})$ was heated under reflux for $3 \mathrm{~h}$, neutralised with acetic acid $(2 \mathrm{M})$ and the resulting precipitate filtered off, washed with water until rinsings were neutral, and dried in vacuo yielding compound 19 as a bright yellow powder (63 mg, $0.144 \mathrm{mmol}, 85 \%), \mathrm{mp} 286-288^{\circ} \mathrm{C} . \mathrm{IR}\left(\mathrm{v} \max , \mathrm{cm}^{-1}\right)$ : 3230, 1592, 1530, 1486, 1277, 1163, 1092, 1014, $887,753$. 
UV/Vis $\left(\lambda\right.$ max, nm, $\left.\varepsilon, \mathrm{cm}^{-1} \mathrm{M}^{-1}\right): 233(36,300), 269$ (21,900), $392(36,300) .{ }^{1} \mathrm{H}$ NMR $\left(300 \mathrm{MHz}, \mathrm{DMSO}-d_{6}\right): \delta_{H}$ $3.86(3 \mathrm{H}, \mathrm{s}, \mathrm{OMe}), 7.00(1 \mathrm{H}, \mathrm{s}, \mathrm{H} 5), 7.38(2 \mathrm{H}, \mathrm{d}, J 8.7 \mathrm{~Hz}, \operatorname{aryl}), 7.41(1 \mathrm{H}, \mathrm{d}, J 2.6 \mathrm{~Hz}, \mathrm{H} 2), 7.53(2 \mathrm{H}, \mathrm{d}, J 8.7 \mathrm{~Hz}$, aryl), $7.67(2 \mathrm{H}, \mathrm{d}, J 8.6 \mathrm{~Hz}$, aryl), $7.87(1 \mathrm{H}, \mathrm{d}, J 0.8 \mathrm{~Hz}, \mathrm{H} 8), 8.00(2 \mathrm{H}, \mathrm{d}, J 8.6 \mathrm{~Hz}, \operatorname{aryl}), 12.01(1 \mathrm{H}, \mathrm{d}, J 1.5 \mathrm{~Hz}, \mathrm{NH})$. ${ }^{13} \mathrm{C}$ NMR (75 MHz, DMSO- $d_{6}$ ): $\delta$ c 56.0 (OMe), 86.7 (C5), 107.8 and 112.0 (aryl C), 116.3 (C8), 117.9 (aryl C), 122.9 (C2), 127.8 and 129.1 (aryl CH), 130.1 and 130.6 (aryl C), 131.1 and 131.4 (aryl CH), 135.1, 136.7, 137.7, 150.0, 156.0 and 157.3 (aryl C), 181.0 (CO). MS (+El, m/z, \%): $437\left(100, \mathrm{MH}^{+}\right), 325$ (18). Anal. calcd for $\mathrm{C}_{24} \mathrm{H}_{15} \mathrm{Cl}_{2} \mathrm{NO}_{3} .0 .5 \mathrm{H}_{2} \mathrm{O}: \mathrm{C}, 64.73 ; \mathrm{H}, 3.62 ; \mathrm{N}, 3.15$. Found: $\mathrm{C}, 64.91 ; \mathrm{H}, 3.40 ; \mathrm{N}, 3.10 \%$.

1-[3-(4-Chlorophenyl)-4-methoxyfurano[2,3-g]indol-7-yl]ethanone (20). A mixture of indole 15 (60 mg, 0.117 $\mathrm{mmol}$ ) and crushed potassium hydroxide (136 mg, $2.34 \mathrm{mmol}$ ) in methanol ( $3 \mathrm{~mL}$ ) was heated under reflux for $3 \mathrm{~h}$, neutralised with acetic acid $(2 \mathrm{M})$, and the resulting precipitate filtered off, washed with water until rinsings were neutral, and dried in vacuo yielding compound 20 as a pale brown powder (36 mg, $0.106 \mathrm{mmol}$, 91\%), $\mathrm{mp}\left(\mathrm{CH}_{2} \mathrm{Cl}_{2}\right) 280-284{ }^{\circ} \mathrm{C}$. IR ( $\left.\mathrm{v} \max , \mathrm{cm}^{-1}\right): 3276,1634,1544,1486,1355,1279,1160,1127,938,838$. UV/Vis $\left(\lambda \max , \mathrm{nm}, \varepsilon, \mathrm{cm}^{-1} \mathrm{M}^{-1}\right): 233(30,200), 358(20,400) .{ }^{1} \mathrm{H}$ NMR $\left(300 \mathrm{MHz}, \mathrm{DMSO}-d_{6}\right): \delta_{\mathrm{H}} 2.51(3 \mathrm{H}, \mathrm{s}, \mathrm{Me})$, $3.83(3 \mathrm{H}, \mathrm{s}, \mathrm{OMe}), 6.94(1 \mathrm{H}, \mathrm{s}, \mathrm{H} 5), 7.37(2 \mathrm{H}, \mathrm{d}, J 8.3 \mathrm{~Hz}$, aryl), $7.41(1 \mathrm{H}, \mathrm{s}, \mathrm{H} 2), 7.53(2 \mathrm{H}, \mathrm{d}, J 8.3 \mathrm{~Hz}, \operatorname{aryl}), 7.85$ $(1 \mathrm{H}, \mathrm{s}, \mathrm{H} 8), 12.03\left(1 \mathrm{H}, \mathrm{br}\right.$ s, NH). ${ }^{13} \mathrm{C}$ NMR $\left(75 \mathrm{MHz}, \mathrm{DMSO}-d_{6}\right): \delta_{\mathrm{c}} 26.4(\mathrm{Me}), 55.9$ (OMe), 86.7 (C5), 107.5 and 112.0 (aryl C), 112.4 (C8), 117.8 (aryl C), 122.8 (C2), 127.8 (aryl CH), 130.2 and 130.6 (aryl C), $131.4(\operatorname{aryl~CH})$, 135.2, 150.8, 155.1 and 156.7 (aryl C), 186.4 (CO). MS (+El, m/z, \%): 341 (100, $\mathrm{MH}^{+}$). Anal. calcd for $\mathrm{C}_{19} \mathrm{H}_{14} \mathrm{ClNO}_{3} .0 .25 \mathrm{CH}_{2} \mathrm{Cl}_{2}: \mathrm{C}, 64.05 ; \mathrm{H}, 4.05 ; \mathrm{N}, 3.88$. Found: $\mathrm{C}, 63.92 ; \mathrm{H}, 4.04 ; \mathrm{N}, 3.88 \%$.

[3-(4-Chlorophenyl)-4-methoxyfurano[2,3-g]indol-7-yl](phenyl)methanol (22). A mixture of indole 18 (60 mg, $0.149 \mathrm{mmol}$ ) and sodium borohydride $(56.4 \mathrm{mg}, 1.49 \mathrm{mmol}$ ) was heated under reflux in ethanol ( $3 \mathrm{~mL})$ for $1 \mathrm{~h}$ and solvent removed in vacuo. The residue was suspended in water $(10 \mathrm{~mL})$, filtered off, washed with water until rinsings were neutral yielding compound 22 as a yellow powder $(0.57 \mathrm{mg}, 0.141 \mathrm{mmol}, 95 \%), \mathrm{mp} 82-84$ ${ }^{\circ} \mathrm{C}$. IR (v max, cm $\left.{ }^{-1}\right)$ : 3425, 2942, 1642, 1601, 1489, 1399, 1194, 1110, 1015, 787, 696. UV/Vis $\left(\lambda \mathrm{max}, \mathrm{nm}, \varepsilon, \mathrm{cm}^{-}\right.$ $\left.{ }^{1} \mathrm{M}^{-1}\right): 236(37,200), 298(23,400) .{ }^{1} \mathrm{H}$ NMR $\left(300 \mathrm{MHz}, \mathrm{DMSO}-d_{6}\right): \delta_{\mathrm{H}} 3.73(3 \mathrm{H}, \mathrm{s}, \mathrm{OMe}), 5.82(1 \mathrm{H}, \mathrm{d}, J 4.5 \mathrm{~Hz}$, $\mathrm{CHOH}), 6.11(1 \mathrm{H}, \mathrm{s}, J 4.5 \mathrm{~Hz}, \mathrm{OH}), 6.69(1 \mathrm{H}, \mathrm{s} . \mathrm{H} 8), 6.79(1 \mathrm{H}, \mathrm{s}, \mathrm{H} 5), 7.27(1 \mathrm{H}, \mathrm{s}, \mathrm{H} 2), 7.33-7.52(9 \mathrm{H}, \mathrm{m}, \operatorname{aryl})$, $11.67\left(1 \mathrm{H}, \mathrm{br}\right.$ s, NH). ${ }^{13} \mathrm{C}$ NMR $\left(75 \mathrm{MHz}, \mathrm{DMSO}-d_{6}\right): \delta$ c 55.6 (OMe), $69.2(\mathrm{CHOH}), 87.0$ (C5), 101.2 (C8), 107.0, 111.3 and 117.1 (aryl C), 122.1 (C2), 127.0, 127.6, 127.7 and 128.5 (aryl CH), 130.0 and 130.2 (aryl C), 131.3 $(\operatorname{aryl} \mathrm{CH}), 137.7,142.9,152.4,152.8$ and $157.6(\operatorname{aryl~C}) . \mathrm{MS}(+\mathrm{El}, \mathrm{m} / \mathrm{z}, \%): 402\left(23,[\mathrm{M}-\mathrm{H}]^{+}\right), 386\left(100,[\mathrm{M}-\mathrm{OH}]^{+}\right)$. Anal. calcd for $\mathrm{C}_{24} \mathrm{H}_{18} \mathrm{ClNO}_{3} .0 .5 \mathrm{H}_{2} \mathrm{O}: \mathrm{C}, 69.82 ; \mathrm{H}, 4.64 ; \mathrm{N}, 3.39$. Found: $\mathrm{C}, 69.73 ; \mathrm{H}, 4.69 ; \mathrm{N}, 3.20 \%$.

1-[3-(4-Chlorophenyl)-4-methoxyfurano[2,3-g]indol-7-yl]ethanol (23). A mixture of indole 20 (80 mg, 0.235 $\mathrm{mmol}$ ) and sodium borohydride ( $89 \mathrm{mg}, 2.35 \mathrm{mmol}$ ) was heated under reflux in ethanol $(4 \mathrm{~mL})$ for $1 \mathrm{~h}$ and the solvent removed in vacuo. The residue was suspended in water $(15 \mathrm{~mL})$, filtered off, washed with water until rinsings were neutral yielding compound 23 as a white powder (78 mg, $0.227 \mathrm{mmol}, 97 \%), \mathrm{mp} 223-225{ }^{\circ} \mathrm{C}$. IR (v max, cm ${ }^{-1}$ ): 3485, 3240, 2980, 2821, 1643, 1488, 1403, 1338, 1198, 1130, 1091, 1067, 1014, 793. UV/Vis $(\lambda$ $\left.\max , \mathrm{nm}, \varepsilon, \mathrm{cm}^{-1} \mathrm{M}^{-1}\right): 236$ (37,200), $296(20,000) .{ }^{1} \mathrm{H}$ NMR (300 MHz, DMSO-d $): \delta_{н} 1.46(3 \mathrm{H}, \mathrm{d}, J 6.4 \mathrm{~Hz}, \mathrm{Me})$, $3.76(3 \mathrm{H}, \mathrm{s}, \mathrm{OMe}), 4.79-4.87(1 \mathrm{H}, \mathrm{m}, \mathrm{C} \underline{\mathrm{HOH}}), 5.37$ (1H, d, J $5.3 \mathrm{~Hz}, \mathrm{CHO} \underline{\mathrm{H}}), 6.79(1 \mathrm{H}, \mathrm{s}, \mathrm{H} 5), 6.82(1 \mathrm{H}, \mathrm{s}, \mathrm{H} 2), 7.30$ $(1 \mathrm{H}, \mathrm{d}, J 2.6 \mathrm{~Hz}, \mathrm{H} 8), 7.35(2 \mathrm{H}, \mathrm{d}, J 8.7 \mathrm{~Hz}, \operatorname{aryl}), 7.53(2 \mathrm{H}, \mathrm{d}, J 8.7 \mathrm{~Hz}, \operatorname{aryl}), 11.70\left(1 \mathrm{H}, \mathrm{br}\right.$ s, NH). ${ }^{13} \mathrm{C}$ NMR $(75$ $\left.\mathrm{MHz}, \mathrm{DMSO}-d_{6}\right): \delta$ c $22.5(\mathrm{Me}), 55.6(\mathrm{OMe}), 62.7(\mathrm{CHOH}), 87.0(\mathrm{C} 5), 99.2(\mathrm{C} 8), 107.1,111.2$ and 117.1 (aryl C), 122.1 (C2), 127.7 (aryl CH), 130.1 and 130.2 (aryl C), 131.3 (aryl CH), 135.8, 152.3, 152.4 and 159.3 (aryl C). MS (+El, $\mathrm{m} / z, \%): 340\left(8,[\mathrm{M}-\mathrm{H}]^{+}\right), 324\left(100,[\mathrm{M}-\mathrm{OH}]^{+}\right)$. Anal. calcd for $\mathrm{C}_{19} \mathrm{H}_{16} \mathrm{ClNO}_{3}: \mathrm{C}, 66.76 ; \mathrm{H}, 4.72 ; \mathrm{N}, 4.10$. Found: C, 66.37; H, 5.01; N, 3.86\%. 


\section{Acknowledgements}

Financial support from the Australian Research Council is gratefully acknowledged. K.P. also acknowledges receipt of an International Postgraduate Research Scholarship from the Australian Government and UNSW.

\section{References}

1. Chunchatprasert, L.; Rao, K. R. N.; Shannon, P. V. R. J. Chem. Soc., Perkin Trans. 1 1992, 1779-83. https://doi.org/10.1039/P19920001779

2. Rao, N. K. R.; Stables, J. N.; Shannon, P. V. R.; Rao, N. K. R.; Chunchatprasert, L. WO. Patent 9402483, 1994.

3. Knölker, H-J.; Fröhner, W. Tetrahedron Lett. 1996, 37, 9183-9186. https://doi.org/10.1016/S0040-4039(96)02181-8

4. Hagiwara, H.; Choshi, T.; Fujimoto, H.; Sugino, E.; Hibino, S. Chem. Pharm. Bull. 1998, 46, 1948-1949. https://doi.org/10.1248/cpb.46.1948

5. Soos, T.; Timari, G.; Hajos, G. Tetrahedron Lett. 1999, 40, 8607-8609.

https://doi.org/10.1016/S0040-4039(99)01803-1

6. Knölker, H-J. ; Fröhner, W. Synthesis 2000, 2131-2136. https://doi.org/10.1055/s-2000-8714

7. Hagiwara, H.; Choshi, T.; Nobuhiro, J.; Fujimoto, H.; Hibino, S. Chem. Pharm. Bull. 2001, 49, 881-886. https://doi.org/10.1248/cpb.49.881

8. Yasuhara, A.; Suzuki, N.; Sakamoto, T. Chem. Pharm. Bull. 2002, 50, 143-145. https://doi.org/10.1248/cpb.50.143

9. Knölker, H-J.; Krahl, M. P. Synlett 2004, 528-530. https://doi.org/10.1055/s-2004-815417

10. Fröhner, W.; Krahl, M. P. ; Reddy, K. R.; Knölker, H-J. Heterocycles 2004, 63, 2393-2407. https://doi.org/10.3987/REV-04-585

11. Krahl, M. P.; Schmidt, A. W.; Knölker, H-J. Heterocycles 2012, 86, 357-370. https://doi.org/10.3987/COM-12-S(N)24

12. Spindler, B.; Kataeva, O.; Knölker, H-J. J. Org. Chem. 2018, 83, 15136-15143. https://doi.org/10.1021/acs.joc.8b02426

13. Pchalek, K.; Condie, G. C.; Kumar, N.; Black, D. StC. Arkivoc 2021, (viii), 1-12. https://doi.org/10.24820/ark.5550190.p011.405

14. Black, D. StC.; Channon, M. F.; Clayton, K. A.; Condie, G. C.; Harper, J. B.; Kumar, N.; Pchalek, K.; Wahyuningsih, T. D. Arkivoc 2006, vii, 67-75. https://doi.org/10.3998/ark.5550190.0007.707

15. Burgstahler, A. W.; Worden, L. R. Org. Synth. 1966, 46, 28-31. https://doi.org/10.15227/orgsyn.046.0028

16. Black, D. StC.; Rezaie, R. Tetrahedron Lett. 1999, 40, 4251-4254. https://doi.org/10.1016/S0040-4039(99)00699-1 